\title{
Application of Wang-Yu Algorithm in the Geometric Constraint Problem
}

\section{Wenhui Li ${ }^{1}$}

Department of Computer Science and Technology, Jilin University

Changchun, 130012, China

E-mail: Iiwh@jlu.edu.cn

\section{Mingyu Sun ${ }^{2}$}

Department of Computer Science and Technology, Jilin University

Changchun, 130012, China,

E-mail:sunmingyu370@sohu.com

Chunhong $\mathrm{Cao}^{3}$

College of Information Science and Engineering, Northeastern University

Shenyang, 110819, China

E-mail:caochunhong@mail.neu.edu.cn

\section{Mingyan Sun}

Changchun West New Economic-Technological Development Zone Construction Industries Co., Ltd Changchun, 130012, China,

E-mail:smy_12130foxmail.com

\section{Honghao Shen}

Changchun West New Economic-Technological Development Zone Construction Industries Co., Ltd Changchun, 130012, China

E-mail:shen1260163.com

The geometric constraint problem is equivalent to the problem of solving a set of nonlinear equations substantially; thus the constraint problem can be transformed to an optimization problem. Inspired by the thought of netting fish in our daily life, Wang-Yu Algorithm starts from the most basic characteristics of the random exploring optimization and ties up two special kinds of data-nets firstly in computer by using the tactics of random moving together; then Wang-Yu Algorithm realizes the object of random exploring optimization from a new direction by netting orderly in the all range of exploring and promptly observing the situation after netting. The experiment shows that it can improve the geometric constraint solving efficiency and possess better convergence property than the compared algorithms.

CENet2015

12-13 September 2015

Shanghai, China

\footnotetext{
${ }^{1}$ Speaker

${ }^{2}$ Correspoding Author

${ }^{3}$ This work is supported by "Grant from Jilin Planned Projects for Science Technology Development” (Grant Nos. 20140101181JC and 20120305).
} 


\section{Introduction}

With the development and popularization of the computer technology, the computer-aided design and manufacturing (CAD/CAM) technology has also been rapidly developed. The $\mathrm{CAD} / \mathrm{CAM}$ technology development and application level has become an important symbol of a country's level of modernization. The geometric constraint solving problem is the current hot issue in the constraint-based design research. A constraint describes the relationship that should be met. Once the user has defined a series of relations and then modifies the parameters, the system will automatically select the appropriate state to satisfy the constraint. This way of thinking is called constraint-based model. Today, many scholars both at home and abroad using the numerical calculation theory, artificial intelligence theory, graph theory and theory of freedom analysis, etc. have studied the constraint solving in-depth. To sum up, it mainly includes the integrated solution method, sparse matrix method, connection analysis method, statute construction method, constraint propagation method, symbolic algebra method and auxiliary line method, etc.[1].

With the deepening of the application, people have found that: the traditional genetic algorithm in the face of complex and large-scale, is not known any general questions about the characteristics of the best one in advance because the search scope is very large, the convergence speed of genetic algorithm is very slow. Due to the role of crossover and mutation operator, some excellent gene fragments are prematurely lost, which has thus limited the scope of the search, arising the premature convergence, so that the search only to find the optimal value in the local area [2]. Thus the various forms of transformation on the traditional genetic algorithm (GA) has aroused great interest of many scientific and technological workers. Great improvements have been achieved based on the traditional genetic algorithm[3-6]; nevertheless, no matter how to change, the main idea of random search of the genetic algorithm optimization has not been changed and the key still lies in the selection, crossover and mutation operations to generate new populations of chromosomes to evaluate and compare the extracted chromosomes, thus to look for excellent target[7]. In the three operations, the optimization speed and effectiveness depend on the crossover operation to a large extent.

As the choice operates only in the original chromosome, of course, it is impossible to produce a better chromosome. The mutation operation only does random sampling throughout the feasible set. Only the crossover operation stipulates to find new chromosomes between the two original optimum chromosomes and it uses a premise: to assume that the possibility of a new superior between the two optimum chromosomes is larger than elsewhere (many cases do so). The crossover operation of this key naturally became a distinctive feature of the genetic algorithm. When the optimization coincides with the prior assumptions, the genetic algorithm will fully demonstrate its advantages; however, when there is a certain gap between problems and presupposes, the genetic algorithm will be very bloated and produce misleading phenomenon. It can be seen that the genetic algorithm still lacks a general and universal applicability in the field of random search optimization algorithm. It is still more appropriate algorithm about part of the optimization problems. When we faced problems that we cannot know any characteristics of the best in advance, it is necessary to look for a more generalized random search optimization algorithm rather than the genetic algorithm.

Inspired by the people's daily fishing thinking and in combination with the advantages of modern computer work, starting from the basic characteristics of the random search optimization, this paper proposes a new algorithm that is, Wang-Yu Algorithm. Firstly, two specific data network (one big and one small) should be woven on the computer[8]. Through an orderly and reasonable woven net, we should timely observe the dynamic of the net. We can use this algorithm to solve geometric constraint problems. With the lessons of premature absorbed from the genetic algorithm, the Wang-Yu Algorithm can achieve the goal of better random search optimization from a new angle. The algorithm net is full of fish from a random search optimization algorithm of the basic characteristics, the correlation random strategy, on the computer first woven two classes (a large and a small) in the so-called special data network. 
Then Cast a net into the search scope of an orderly, reasonable and timely dynamic observation net. It would be better to achieve the goal of random search optimization from a new aspect.

\section{Geometric Constraint Solving}

From the artificial intelligence point of view [9-10], the nature of the design problem is a constraint satisfaction problem. The ultimate goal of solving a geometric constraint problem is to determine the specific coordinates of the location of each in the geometry.

Usually, the constraint problem can be formalized as $(E, C)[11]$, where $E=\left(e_{1}, e_{2}, \ldots, e_{\mathrm{n}}\right)$, represents geometric elements, such as points, lines, circles, etc.; $C=\left(c_{1}, c_{2}, \ldots, c_{m}\right), c_{i}$ represents the added constraints between these geometric elements. Generally a constraint corresponds to an algebraic equation, so the constraint can be expressed as

$$
\begin{gathered}
f_{1}\left(x_{0}, x_{1}, x_{2}, \ldots, x_{n}\right)=0 \\
\ldots \\
f_{m}\left(x_{0}, x_{1}, x_{2}, \ldots, x_{n}\right)=0
\end{gathered}
$$

$X=\left(x_{0}, x_{1}, \ldots, x_{n}\right), x_{\mathrm{i}}$ is the value of some parameters of the geometric elements $e_{i}$, for example the two-dimensional point can be expressed as $\left(x_{1}, x_{2}\right)$.Constraint solving is to find the $X$ that satisfies(1).

$$
F\left(X_{i}\right)=\sum_{i=1}^{m}\left|f_{i}\right|
$$

Obviously if $X_{j}$ meet $F\left(X_{j}\right)=0$, then $X_{j}$ satisfies Equation (2.1); therefore, the constraint solving problem can be transformed into an optimization problem, which only needs $\min F\left(X_{j}\right)$ $<$, is a certain threshold. In order to improve the speed of the algorithm, we use the absolute value of $f_{i}$ rather than the sum of squares to represent the constraint equations.

With Equation (2.2) and the min $F\left(X_{j}\right)<$, which is get through our Wang-Yu Algorithm know, does not require that $m=n$; thus it can solve under-constrained and over-constrained problem.

\section{Network Fish Algorithm}

\subsection{Main Idea of the Wang-Yu Algorithm}

As the random search optimization algorithm will mainly face complex and large-scale general questions, and do not know any main features about the best ones in advance, the design ideas of Wang-Yu Algorithm will highlight the following two points.

Firstly, during the entire random search optimization process, all feasible points taken from the evaluation feature certain degree of randomness, and in sufficient quantity, also the whole distribution of all evaluated and possible points should get balanced. This optimization results can be widely representative and it can prevent the misleading phenomenon from the root.

Secondly, during the whole random search optimization operation, it make full use of existing information generated from the optimization process provided by the problem itself so as to find the optimal target as soon as possible. It is the key to improve work efficiency and optimization accuracy. 


\subsection{Specific Steps to Realize Wang-Yu Algorithm}

Based on the above two points, the specific steps of the Wang-Yu algorithm is described in Fig. 1.

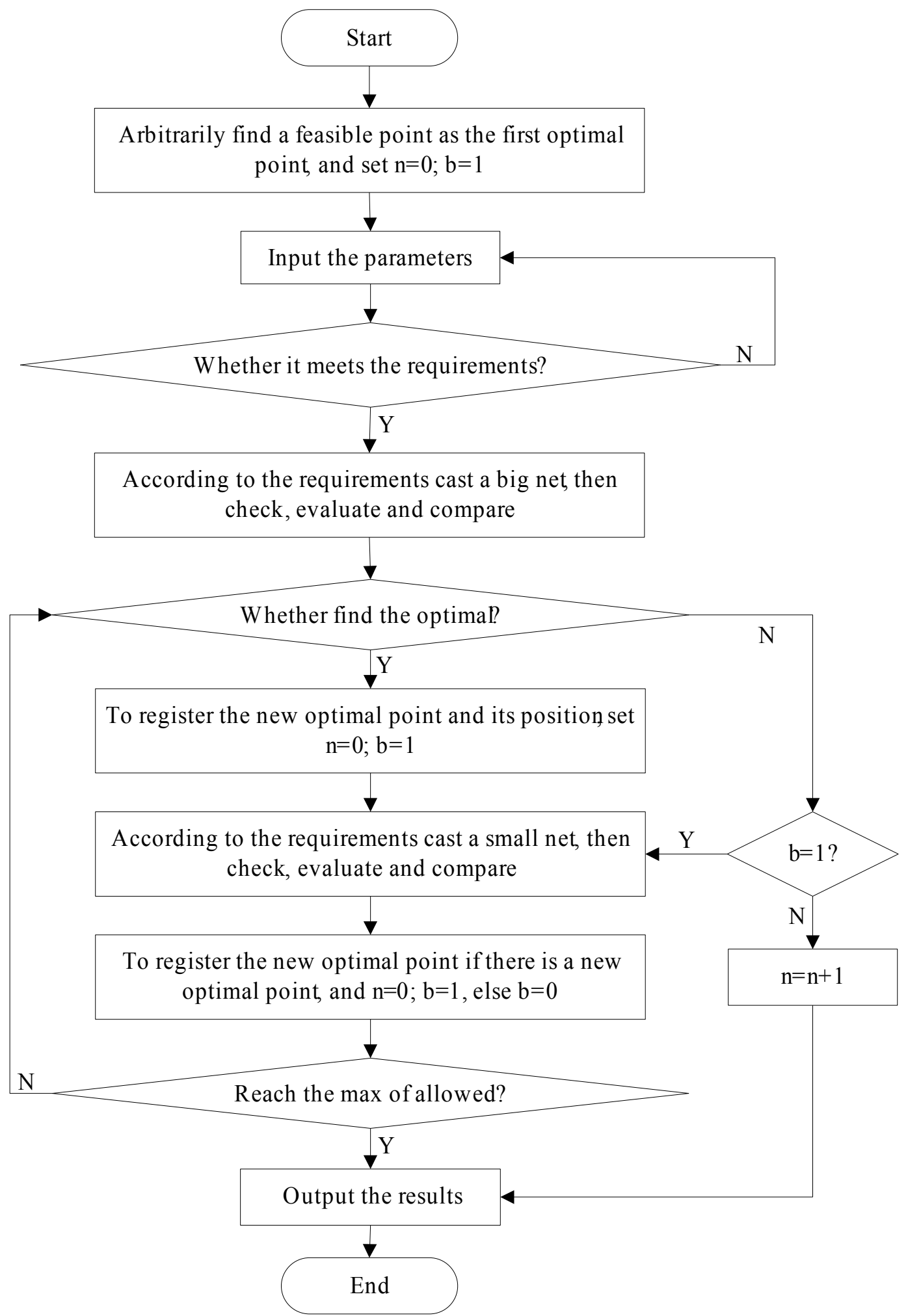

Figure 1: Wang- Yu Algorithm Flowchart 


\section{Parameters Introduction}

The number of net knots of both large net and small net is $w_{1}^{*} w_{2}{ }^{*} w_{3}$, the total length of each dimension of the search for a small net is $\mathrm{R}^{*}\left(1 / w_{l}\right)$ (R times the step length $\left(1 / w_{1}\right)$ in each dimension of a large net), $\mathrm{Q}$ is the maximum allowable number of large net continuous failure times, $\mathrm{C}$ is the total number of the extracted "feasible point" of the entire optimization process.

(1) Scale of small net parameter: small net's fixed "step size" $\left(\mathrm{XL}_{1}, \mathrm{XL}_{2} \ldots \ldots \mathrm{XL}_{\mathrm{n}}\right)$ for each dimension (assuming that n-dimensional) and its number of each dimension's "Step point" $\left(\mathrm{XW}_{1}, \mathrm{XW}_{2} \ldots \ldots \mathrm{XW}_{\mathrm{n}}\right)$ ("Step point" is the aliquots of boundaries of each dimension, "step size" is the distance on the same dimension between two adjacent "Step point" or the length of aliquots of a part).

(2) Scale of large net parameter: large net's number of each dimension's (assumed that ndimensional) "step point" $\left(\mathrm{DW}_{1}, \mathrm{DW}_{2} \ldots \ldots \mathrm{DW}_{\mathrm{n}}\right)$.

(3) Termination condition parameters: the total number of large net needs to be casted to find at least one point in the global optimum of "cronies point".

\section{Experiment Results of Wang-Yu Algorithm}

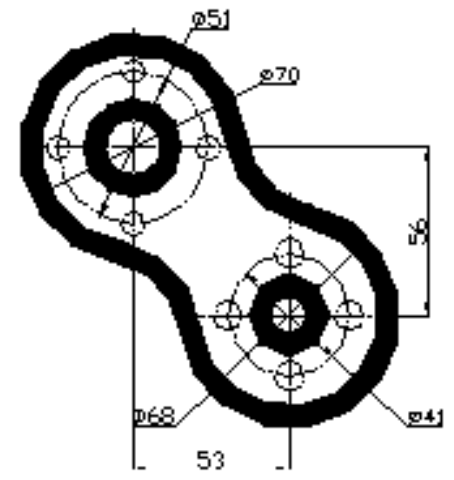

(a) the original design figure

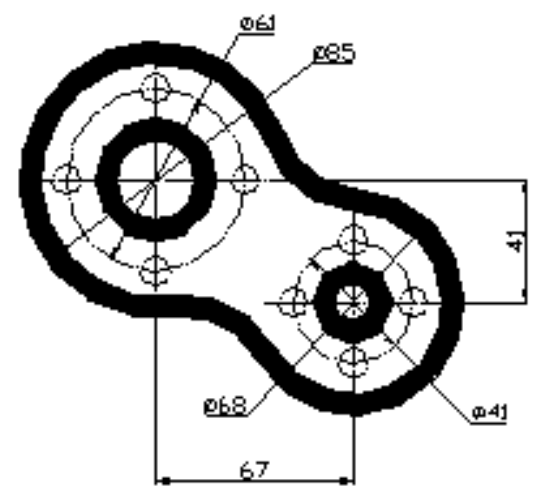

(b) the variable design figure

Figure 2: Sketch Design Figure and New Graphics with the Wang-Yu Algorithm

As is shown in Fig. 2, (a) is the original design figure and (b) is the new figure whose part of the size or angle has changed from the use of Wang-Yu algorithm. Here $w_{1}{ }^{*} w_{2}{ }^{*} w_{3}$ is $100, \mathrm{R}$ is $0.1, \mathrm{Q}$ is 2 . It can be seen from the above figure that after the users have drawn the picture, you can modify the size value at any time. Systems realized through the Wang-Yu algorithm will update itself in accordance with the new size value timely, making it easier to achieve the creation of graphic changes and a series of parts.

\begin{tabular}{|c|c|c|c|} 
Algorithm & $\begin{array}{c}\text { Evolution } \\
\text { algebra }\end{array}$ & $\begin{array}{c}\text { Take up cpu } \\
\text { time(s) }\end{array}$ & $\begin{array}{c}\text { Appear the best solutions of } \\
\text { algebraic }\end{array}$ \\
\hline GA & 300 & 200 & 169 \\
\hline PSO & 20 & 40 & 15 \\
\hline Wang-Yu & 14 & 20 & 10 \\
\hline
\end{tabular}

Table 1: Experimental Results Comparison among GA, PSO and Wang-Yu

In this experiment, it costs 200 seconds when using the optimal results resulting from the genetic algorithms and 40 seconds when using the optimal results resulting from PSO; however, it costs only 20 seconds when using Wang-Yu algorithm, which indicates that: Wang-Yu algorithm can use the main time directly for "feasible point" test, evaluation and comparison and it costs meager time for other ancillary work while compared with the genetic algorithm with almost no duplication of work. As Wang-Yu algorithm uses a randomly-selected point 
linkage strategy, it can not only ensure the randomness but also take care of the points' balance distribution. It is because of the sharp reduction in the number of random function as called, the Wang-Yu algorithm will show such excellent performance.It can be seen from Table 1 that geometric constraint solving based on the Wang-Yu algorithm can get better performance and better convergence in relation to the contrast algorithm.

\section{Conclusion}

The geometric constraint solving problem has always been the core content of the parametric design. Whether it is to solve geometric constraint problems well or not is the key of parametric design system. In this paper, we transform the constraint equations of the geometric constraint problem into the optimization model so that the constraint solving problem is transformed into the optimization problem.

The Wang-Yu algorithm penetrates the essence of random search optimization algorithm to draw on the random advantages of genetic algorithm with lessons of premature from the genetic algorithm, and it has better achieved the goal of the random search optimization from a new angle. The experimental results of Wang-Yu algorithm for geometric constraint solution show that: during the process of solving optimization problems, when we don't know any of the characteristics of the best one in advance, the Wang-Yu algorithm is more general than the genetic algorithm and has a broader adaption.

\section{References}

[1] Yuan Bo. The Research and Implement of Geometric Constraint Solving [D]. Tsinghua University, Beijing, 1999. (In Chinese)

[2] Xiao Wei. Some Improvement of the Genetic Algorithm [J]. Computer Engineering and Application, 2004, 49(4):53-56. (In Chinese)

[3] Ling Yingbiao. Genetic Algorithm for Solving SAT Problems Based on Learning Clause Weights [J].Chinese Journal of Computers, 2005, 28(9):1477-1481. (In Chinese)

[4] Zhang Ling, Zhang Bo. Good Point Set Based Genetic Algorithm [J].Chinese Journal of Computers, 2001, 24(9):917-922. (In Chinese)

[5] Liu Xichun, Yu Shouyi. A Genetic Algorithm with Fast Local Adjustment [J].Chinese Journal of Computers, 2006, 29(1): 100-105. (In Chinese)

[6] Liu Lifang, Huo Hongwei, Wang Baoshu. PHGA-COFFEE: Aligning Multiple Sequences by Parallel Hybrid Genetic Algorithm [J]. Chinese Journal of Computers 2006, 29(5): 727-733. (In Chinese)

[7] Liu Baoding, Zhao Ruiqing, Wang Gang. Uncertain Programming with Applications [M]. Beijing: Tsinghua University Press, 2003:1-22. (In Chinese)

[8] Fu Guoqing, Xu Weixiang, Li Xiaozheng. A Random Exploring Optimization Algorithm: WangYu Algorithm [J]. Journal of Beijing Jiaotong University, 2007, 31(6):123-127. (In Chinese)

[9] Fu Jingsun, Cai Zixing, Xu Guangyou. Artificial Intelligence and its Applications [M]. Beijing: Higher Education Press, 1987: 15-28. (In Chinese)

[10]Zhou Ji, Cha Jianzhong, Xiao Renbin, Intelligent Design [M]. Beijing: Higher Education Press, 1998: 1-12. (In Chinese)

[11] Liu Shengli, Tang Min, Dong Jinxiang. Two Spatial Constraint Solving Algorithms [J]. Journal of Computer-aided Design \& Computer Graphics, 2003, 15(8): 1011-1029. (In Chinese) 